\title{
To the Problems of Cleaning of Hard-grades Raw Cotton
}

\section{Rosulov Rk ${ }^{1 *}$ and Saphoyev AA}

Tashkent Institute of Textile and Light Industry, Tashkent, Uzbekistan

\begin{abstract}
Questions of optimization of modes of clearing of a cotton taking into account selection grades is one of the primary goals in a cotton of the cleaning industry, especially at a choice according to plan cotton cleanings. The basic lack of cleaners of the cotton, applied now, is invariable operating modes of working bodies without initial quality indicators of cleared cotton. Relative reduction of the sizes of seeds at new selection grades of cotton that it is necessary to consider at their processing on cotton of cleaners is installed. For infringement of monotony of process of clearing of cotton it is recommended to alternate polytypic working bodies. Possibility of application of flexible technological systems which it is successfully used in various branches of mechanical engineering, and for flexible clearing of a cotton-raw of weed impurity is considered.
\end{abstract}

Keywords: Raw cotton; Cleaning effect; Cotton seed; Cotton peat cotton ginning industry; Cleanup plan; Small litter cleaner; Cleaner large litter

\section{Introduction}

In recent years, in the Republic of Uzbekistan is grown over 10 zoned breeding sorts of cotton, of which most widely used are selected sorts as Bukhara-6 Namangan-77 and C-6524. Therefore, the problems of optimization of ginning with the selection of sorts are one of the main tasks in the cotton gaining industry. To develop nig wowing out the cotton fiber content of vices and trash within the established settlement rules, processing of raw cotton is carried out on a definite plan treatment. Choice plans of ginning is made depending on its initial infestation, welling and commercial grade, in conjunction with subsequent purification of cotton fiber.

As is known, the main technological equipment for cleaning cotton, currently cleaners are small litter type $1 \mathrm{HK}$ and a cotton plant type UHK. Using of these machines in the cotton industry has contributed, in principle, the production of cotton fiber with the required quality [1]. The main working body of the cleaner small litter type $1 \mathrm{HK}$ Peg drum diameter is $400 \mathrm{~mm}$, which number in the cleaner is eight pieces, and the main working body cleaner type UHK is as Peg drums, which number in the cleaner is six pieces, and serrated drums diameter 480 $\mathrm{mm}$, the number of which cleaner is also six.

Principle of operation and Peg saw drums based in their shockshaker effects on the feed material (raw cotton) and dragging of them on the grate to remove trash. These drums have different peripheral speeds Peg drum rotates at a peripheral speed of $9 \mathrm{~m} / \mathrm{s}$, and the serrated drum rotates at a circumferential speed of $7 \mathrm{~m} / \mathrm{s}$.

In general, the existing modes of reels of cotton cleaners contribute to a satisfactory cleaning of raw cotton, but the emergence of so-called "difficult clean cotton sort" sort of cotton and in this regard, the sharp decline in the cleaning effect of such machines in the processing of cotton requires finding new opportunities improve the cleaning effect machines.

The main drawback of these machines, in our opinion, is that the cleaning process takes place within them under constant operating conditions of the workers and did not take into account the initial qualitative indicators to be cleansed of cotton, such as breeding and industrial grade, the initial infestation, etc.

At the same time there is a trend of increasing the share of socalled "difficult purified cotton variety" varieties in total collection of raw cotton, which are very difficult to clean because of the structure of the fibers, although they have certain advantages in indicators such as productivity, physical and mechanical properties of the fiber, ripening, etc.

Therefore, in practice there is often a fact- technological machine that works well and provides a high cleaning effect on one breeding varieties of cotton, due to the increased strength of the attachment of the litter to the fiber cannot cope with another cotton varieties of cotton breeding different varieties.

Increase speed modes drum cleaners above specified values leads to a sharp deterioration in the quality of cleaning due to the increase of seed damage and the resulting in such deleterious consequence of this defect as "peel fiber" [2].

Established by the heavy-duty cleaners drums of raw cotton were used for the processing of previously cultivated varieties of cotton industry. At the same time, now recommended for the cultivation of new cotton varieties differ from them a number of indicators, such as yield, ripening, fiber length, seed size, etc.

One major factor that plays an important role in the process ginning is the size of the seed cotton.

In new breeding cotton varieties tend to reduce the size of the seed. Therefore, we have carried out studies to determine the size of seed cotton varieties recommended again by the example of the selection sort "Porlock."

The methodology of the study was as follows: samples were taken by experienced seed quantity of 100 pieces. Each sample was weighed and determined by the total whole sample. Then, using a special device alternately measure the length and width of the seeds, and both the length and width of seeds were divided in turn into three groups and the amount of seeds attributable to a particular group.

Experiments were performed in triplicate and the results are shown in Table 1.

*Corresponding author: Rosulov Ruzimurad Khasanovich, Tashkent Institute of Textile and Light Industry, Tashkent, Uzbekistan, E-mail: rasulov.ruzimurad@mail.ru

Received April 01, 2015; Accepted April 15, 2015; Published May 21, 2015

Citation: Rosulov RK, Saphoyev AA (2015) To the Problems of Cleaning of Hardgrades Raw Cotton. J Textile Sci Eng 5: 192. doi:10.4172/2165-8064.1000192

Copyright: (c) 2015 Rosulov RK, et al. This is an open-access article distributed under the terms of the Creative Commons Attribution License, which permits unrestricted use, distribution, and reproduction in any medium, provided the original author and source are credited. 


\begin{tabular}{|c|c|c|c|c|}
\hline $\begin{array}{c}\text { Weight of the } \\
\text { seed, c. }\end{array}$ & Length, sm. & $\begin{array}{c}\text { The number } \\
\text { of seeds }\end{array}$ & $\begin{array}{c}\text { Seed width in } \\
\text { cross section, sm. }\end{array}$ & $\begin{array}{c}\text { The number } \\
\text { of seeds }\end{array}$ \\
\hline \multirow{3}{*}{10,16} & $0,68-0,72$ & 14 & $0,35-0,40$ & 22 \\
\cline { 2 - 5 } & $0,75-0,81$ & 36 & $0,41-0,45$ & 55 \\
\cline { 2 - 5 } & $0,83-1,0$ & 50 & $0,50-0,55$ & 23 \\
\hline \multirow{3}{*}{10,12} & $0,68-0,72$ & 15 & $0,35-0,40$ & 25 \\
\hline \multirow{3}{*}{10,18} & $0,75-0,81$ & 37 & $0,41-0,45$ & 49 \\
\hline \multirow{3}{*}{$10,83-1,0$} & 48 & $0,50-0,55$ & 26 \\
\hline & $0,68-0,72$ & 37 & $0,35-0,40$ & 36 \\
\hline & $0,75-0,81$ & 23 & $0,41-0,45$ & 38 \\
\hline & $0,83-1,0$ & 40 & $0,50-0,55$ & 24 \\
\hline
\end{tabular}

Table1: The results of measurements of the dimensions of seeds

\begin{tabular}{|c|c|c|c|c|c|c|c|}
\hline \multirow{2}{*}{ No } & \multirow{2}{*}{$\begin{array}{l}\text { Selected } \\
\text { seed sorts }\end{array}$} & \multirow{2}{*}{$\begin{array}{c}\text { industrial } \\
\text { grade }\end{array}$} & \multirow{2}{*}{$\begin{array}{l}\text { The } \\
\text { Class }\end{array}$} & \multirow{2}{*}{$\begin{array}{c}\text { Starting } \\
\text { cloggyd-ness, } \%\end{array}$} & \multicolumn{3}{|c|}{$\begin{array}{l}\text { Clearing effect, } \% \text {, } \\
\text { after: }\end{array}$} \\
\hline & & & & & I-1 HK & UHK & II-1 HK \\
\hline 1 & Namangan-77 & 1 & 1 & 2,40 & 11,9 & 35 & 41,7 \\
\hline 2 & Bukhara-8 & 2 & 1 & 3,8 & 15,8 & 25 & 25 \\
\hline 3 & Bukhara-6 & 1 & 1 & 2,85 & 11,5 & 63,7 & 47,4 \\
\hline
\end{tabular}

Analysis of the experimental results presented in Table 2 shows that seeds $0.68-0.72$ sm length averaging $32 \%$, a length $0,75-0.81$ sm averages $32 \%$ and with a length of $0.83-1,0 \mathrm{sm}-46 \%$ of total seed measured. Also it should be noted that seeds from the cross-section width $0.35-0.40 \mathrm{sm}$ averaging $27.7 \%$, with a width of $0.41-0.45$ averaged $47.3 \%$ and with a width of $0.50-0.55 \mathrm{sm} 25 \%$.

Studies have shown that the size of the seed once again recommended varieties of cotton by about $15-18 \%$ less than that of cotton grown previously.

When one of the - same peripheral speed of the drum shoot the breeze seeds with a relatively large size are arranged more damage than smaller seeds having sizes. Consequently, when cleaning difficult purified cotton variety cotton cultivars with relatively smaller size of the seed can be increased ripper drum peripheral speed, thereby increasing the effect of cleaning machines.

There are recommendations for the multiplicity of ginning on Peg and saw drums for difficult purified cotton variety selection of cotton, for example 1-2 commercial grade raw cotton with an initial blockage in the range $3.6-7.9 \%$ is recommended to use 32 drums and 4 saw drums which greatly complicates the work of cleaning shop ginning in terms of layout and installation of ginning equipment, not to mention of the additional cost of electricity.

We have, in a production environment ginning plant experiments were conducted to study the effect of selected varieties of seed cotton cleaning effect on the process equipment.

In experiments through line ginning, consisting of front wiper fine litter $1 \mathrm{HK}$, ginning machine UHK and rear wiper fine litter $1 \mathrm{HK}$ skip certain batch of raw cotton of different varieties and breeding by sampling after each machine is determined by its cathartic effect.

The results of the experiments were repeated three times are shown in Table 2.

As It shown from the table, in the processing of different selecting and industrial grades and classes of cotton there are different, but equally low cathartic effect, which does not satisfy the cotton industry.

Analyzing the causes of the low cleaning effect of technological machines, was the dependence of the total effect the cleaning wiper with the same type of working bodies of the number of cleaning section, which has the character of a geometric progression and is expressed by the formula;

$$
\hat{E}_{\sum^{m}}=K_{1} \frac{1-q^{m}}{1-q}
$$

Where $\mathrm{K}_{1}$ - cleaning effect of the first section, \%;

$\mathrm{q}$ - The denominator geometric;

$\mathrm{m}$ - Number of cleaning sections progress.

The denominator of the geometric progression is determined through the cleansing effect of the first section:

$$
q=\left(\frac{100-\hat{E}_{1}}{100}\right)^{2}
$$

Analysis of the formula (1) shows that with increasing amounts of the same type working in the cleaner can not drastically improve the cleansing effect. For example, a cleaner $1 \mathrm{HK}$ Peg reel on which is applied the saw ginning factories; cleaning effect gives only $40 \%$, while the cleaning effect of the first section - 7\%.

With an extremely large number of sections, when the total cleaning effect is

$$
\hat{E}_{\sum_{m \rightarrow \infty} m}=K_{1} \frac{1}{1-q}
$$

Putting into this expression $\mathrm{K} 1=7 \%, q=\left(\frac{100-\hat{E}_{1}}{100}\right)^{2}=-0,86$ we get $\hat{E}_{\sum_{m \rightarrow \infty} m}=50 \%$.

Consequently, on the cleaner with Peg a drum for any working sections cannot be obtained cleaning effect is from $50 \%$.

Almost the same number of working bodies in the cleaner is not big, as the monotony of the process not only dramatically reduces the cleaning power of each subsequent section, but also leads to a deterioration of its appearance and the formation of defects.

Thus, to use in a large number of similar working bodies inappropriate. This is confirmed by the experience of the textile industry, where at each stage of processing fibers (loosening, piece and carding) uses a variety of working bodies, and the same machine in a row never set.

In addition, we consider the possibility of flexible manufacturing systems (FMS), which has been successfully used in different branches of engineering, and for flexible ginning depending on the grade, class and debris.

\section{Conclusions}

1. It is determined hat as a relative reduction in the size of seeds in breeding new varieties of cotton; this must be considered in their processing on the cotton gin.

2. For breasting of monotonic process ginning of cotton is recommended to alternate different types working bodies.

\section{References}

1. Primary processing of raw cotton.

2. Miroshnichenko GI (1972) Fundamentals of Machine Design of primary processing of cotton. Note: UHK 1 and $\mathrm{HC}$-cotton clearing machines. 